\title{
Bifurcation of Sound Waves in a Disturbed Fluid
}

\section{Zuwen Qian}

Institute of Acoustics, Chinese Academy of Sciences, Beijing, China

Email address:

qianzw@mail.ioa.ac.cn

\section{To cite this article:}

Zuwen Qian. Bifurcation of Sound Waves in a Disturbed Fluid. American Journal of Modern Physics. Vol. 6, No. 5, 2017, pp. 91-95. doi: 10.11648/j.ajmp.20170605.13

Received: July 12, 2017; Accepted: July 19, 2017; Published: August 15, 2017

\begin{abstract}
An equation that describes the wave propagation in the disturbed medium was deduced from the Lighthill's equation. The so-called perturbation-cumulative approximation was suggested to solve this equation and the period-doubling bifurcation solutions were given. The results obtained in this paper helps to provide insights to the mechanism of the turbulence formation.
\end{abstract}

Keywords: Period-Doubling Bifurcation, Chaos, Subharmonics, Disturbed Media

\section{Introduction}

Many numerical and experimental works (for example, Ref. [1] and Refs. [2, 3]) demonstrated that as the control parameters increase the system can route to chaos through period-doubling bifurcations. In history, the period-doubling vibrations or the period-doubling bifurcations in different practical situations were observed by many physicists one after another, for example, as described in ref. [4], "when a vessel containing liquid is made to vibrate vertically, a pattern of standing waves is often observed at the free surface. These waves were first studied experimentally by Faraday, who noticed that the frequency of the liquid vibrations was only half that of the vessel." A thorough theoretical research to this problem was given by the authors of Ref. [4]. Moreover, the motions of an inverted pendulum or a swing played by children are well known examples of the period-doubling vibrations. Regarding these motions, many theoretical explanations can be found in references (for example, Ref. [5] and the papers cited by it to introduce the analytical solution of inverted pendulum).

About thirty years ago, the authors of the Refs. [6, 7] observed the first subharmonic wave in a disturbed water. Up to date, however, there is no publication that provides any interpretation to the phenomenon. In order to investigate this problem, the author started from the Lighthill's equation, and an equation to describe the wave propagation in the disturbed medium was deduced and the so-called perturbationcumulative approximation was suggested to solve this equation, from which the period-doubling bifurcation solutions were obtained. The results obtained by this paper would help us to research the mechanism of the turbulence formation.

\section{Theory}

For an ideal fluid and neglecting the non-linearity due to the medium, Lighthill stress tensor can be written as [8]

$$
T_{i j}=\rho v_{i} v_{j}
$$

which satisfies Lighthill equation

$$
\frac{1}{C_{0}^{2}} \frac{\partial^{2} \rho}{\partial t^{2}}-\nabla^{2} \rho=\frac{1}{C_{0}^{2}} \frac{\partial^{2} T_{i j}}{\partial x_{i} \partial x_{j}}
$$

where $\rho$ denotes the density of the medium and $v_{i}$ is the $i$ th velocity component of the fluid particles. For one dimensional motion

$$
T_{11}=\rho v^{2}
$$

There is a sound wave propagating in the medium at rest, in which velocity component of the fluid particles can denoted by

$$
v=v_{0} \cos 2(\tau-\sigma), \tau=\omega t, \sigma=k x
$$

where $\omega$ and $k$ are the frequency and acoustic wave-number, 
respectively. If the fluid medium is disturbed at a velocity $V_{0}$, then the velocity component of the fluid particles can be denoted

$$
v=V_{0}+v_{0} \cos 2(\tau-\sigma)
$$

and the corresponding Lighthill stress tensor can be denoted by $T_{11}^{(1)}$, which can denote

$$
T_{11}^{(1)}=\rho\left\{V_{0}^{2}+\frac{1}{2} v_{0}^{2}+2 V_{0} v_{0} \cos 2(\tau-\sigma)+\frac{1}{2} v_{0}^{2} \cos 4(\tau-\sigma)\right\}
$$

Substituting (3) into Lighthill wave equation (2) yields

$$
\frac{\partial^{2} \rho}{\partial \tau^{2}}-\frac{\partial^{2} \rho}{\partial \sigma^{2}}=\varepsilon\left\{g_{1}(\sigma, \tau) \frac{\partial^{2} \rho}{\partial \sigma^{2}}+g_{2}(\sigma, \tau) \frac{\partial \rho}{\partial \sigma}-g_{3}(\sigma, \tau) \rho\right\}
$$

where

$$
\left.\begin{array}{c}
\varepsilon=M m \\
g_{1}(\sigma, \tau)=\frac{M}{m}+\frac{m}{2 M}+2 \cos 2(\tau-\sigma)+\frac{m}{2 M} \cos 4(\tau-\sigma) \\
g_{2}(\sigma, \tau)=8 \sin 2(\tau-\sigma)+4 \frac{m}{M} \sin 4(\tau-\sigma) \\
g_{3}(\sigma, \tau)=8\left[\cos 2(\tau-\sigma)+\frac{m}{M} \cos 4(\tau-\sigma)\right]
\end{array}\right\}
$$

and $M, m$ are the Mach numbers corresponding to the velocity of the flow $V_{0}$ and the amplitude of the sound wave $v_{0}$. For the subsonic flow, they are less than $1 . \mathrm{Mm}$ is a small quantity and the equation (4) can be solved by the perturbation approximation. Let the solution be [9]

$$
\begin{aligned}
\rho=\rho^{(0)}+\varepsilon \rho^{(1)}+\varepsilon^{2} \rho^{(2)}+\ldots \ldots & =\sum_{m_{1}} \varepsilon^{m_{1}} \rho^{\left(m_{1}\right)} \\
& \frac{\partial^{2} \rho^{(1)}}{\partial \tau^{2}}-\frac{\partial^{2} \rho^{(1)}}{\partial \sigma^{2}}=-A_{1}^{(1)} \cos (\tau-\sigma)-A_{3}^{(1)} \cos 3(\tau-\sigma)-A_{5}^{(1)} \cos 5(\tau-\sigma)
\end{aligned}
$$

Substituting (7) into (4) and equating coefficients of like powers of $\varepsilon$ to zero give

$$
\begin{aligned}
& \varepsilon^{0}: \quad \frac{\partial^{2} \rho^{(0)}}{\partial \tau^{2}}-\frac{\partial^{2} \rho^{(0)}}{\partial \sigma^{2}}=0 \\
& \varepsilon^{1}: \quad \frac{\partial^{2} \rho^{(1)}}{\partial \tau^{2}}-\frac{\partial^{2} \rho^{(1)}}{\partial \sigma^{2}}=g_{1}(\sigma, \tau) \frac{\partial^{2} \rho^{(0)}}{\partial \sigma^{2}} \\
& +g_{2}(\sigma, \tau) \frac{\partial \rho^{(0)}}{\partial \sigma}-g_{3}(\sigma, \tau) \rho^{(0)} \\
& \varepsilon^{2}: \quad \frac{\partial^{2} \rho^{(2)}}{\partial \tau^{2}}-\frac{\partial^{2} \rho^{(2)}}{\partial \sigma^{2}}=g_{1}(\sigma, \tau) \frac{\partial^{2} \rho^{(1)}}{\partial \sigma^{2}}+ \\
& g_{2}(\sigma, \tau) \frac{\partial \rho^{(1)}}{\partial \sigma}-g_{3}(\sigma, \tau) \rho^{(1)}
\end{aligned}
$$

$$
\begin{aligned}
& \varepsilon^{m_{1}+1}: \quad \frac{\partial^{2} \rho^{\left(m_{1}+1\right)}}{\partial \tau^{2}}-\frac{\partial^{2} \rho^{\left(m_{1}+1\right)}}{\partial \sigma^{2}}= \\
& g_{1}(\sigma, \tau) \frac{\partial^{2} \rho^{\left(m_{1}\right)}}{\partial \sigma^{2}}+g_{2}(\sigma, \tau) \frac{\partial \rho^{\left(m_{1}\right)}}{\partial \sigma}-g_{3}(\sigma, \tau) \rho^{\left(m_{1}\right)}
\end{aligned}
$$

Obviously, the solutions for equation (8) can be [9]

$$
\rho^{(0)}=\left\{\begin{array}{l}
A_{0} \cos l(\tau-\sigma) \\
B_{0} \sin l(\tau-\sigma),
\end{array} \quad l=1,2,3, \ldots\right.
$$

of which the solutions are either of the period-doubling waves or periodic waves, according as $l$ is odd or even. At first, taking the cosine term when $l=1$ as the zeroth order solution in equation (11) and substituting it into (9) yield

where

$$
\varepsilon A_{1}^{(1)}=\left(M^{2}+\frac{m^{2}}{2}+M m\right) A_{0}, \varepsilon A_{3}^{(1)}=\left(M m+\frac{1}{4} m^{2}\right) A_{0}, \varepsilon A_{5}^{(1)}=\frac{1}{4} m^{2} A_{0}
$$

From the equation (12) it can be seen that the inhomogeneous terms in its right hand correspond to the secular terms, which make the problem to be solved more difficult. In order to find the period solutions in time domain, the cumulative solutions in spatial domain will be sought instead of eliminating the secular terms [10], which can be (cf. Appendix 1)

$$
\rho^{(1)}(\tau, \sigma)=\sum_{n=0}^{2} \frac{A_{2 n+1}^{(1)}}{4}[\cos (2 n+1)(\tau-\sigma)-2(2 n+1) \sigma \sin (2 n+1)(\tau-\sigma)]
$$

where the cumulative term is proportional to $\sigma$. Substituting equation (14) into (10) yields 


$$
\frac{\partial^{2} \rho^{(2)}}{\partial \tau^{2}}-\frac{\partial^{2} \rho^{(2)}}{\partial \sigma^{2}}=\sum_{n=0}^{4} A_{2 n+1}^{(2)} \cos (2 n+1)(\tau-\sigma)+\sum_{n=0}^{4} B_{2 n+1}^{(2)} \sigma \sin (2 n+1)(\tau-\sigma)
$$

where

$$
\begin{gathered}
\mathcal{E}^{2} A_{1}^{(2)}=\frac{1}{4}\left\{\left[3\left(M^{2}+\frac{1}{2} m^{2}\right)-5 M m\right] A_{1}^{(1)}+\left(\frac{11}{9} M m-\frac{13}{36} m^{2}\right) A_{3}^{(1)}+\frac{19}{100} m^{2} A_{5}^{(1)}\right\} \\
\varepsilon^{2} B_{1}^{(2)}=\frac{1}{2}\left\{\left[\left(M^{2}+\frac{1}{2} m^{2}\right)-M m\right] A_{1}^{(1)}+\frac{1}{3}\left(M m-\frac{1}{4} m^{2}\right) A_{3}^{(1)}+\frac{1}{20} m^{2} A_{5}^{(1)}\right\} \\
\varepsilon^{2} A_{3}^{(2)}=\frac{3}{4}\left\{\left(M m-\frac{7}{4} m^{2}\right) A_{1}^{(1)}+A_{3}^{(1)}\left(M^{2}+\frac{1}{2} m^{2}\right)+\frac{17}{25} M m A_{5}^{(1)}\right\} \\
\varepsilon^{2} B_{3}^{(2)}=\frac{3}{2}\left\{3\left(M m-\frac{1}{4} m^{2}\right) A_{1}^{(1)}+A_{3}^{(1)}\left(M^{2}+\frac{1}{2} m^{2}\right)+\frac{3}{5} M m A_{5}^{(1)}\right\} \\
\varepsilon^{2} A_{5}^{(2)}=-\frac{5}{16} m^{2} A_{1}^{(1)}+\frac{35}{36} M m A_{3}^{(1)}+\frac{3}{4}\left(M^{2}+\frac{1}{2} m^{2}\right) A_{5}^{(1)} \\
\varepsilon^{2} B_{5}^{(2)}=\frac{5}{2}\left\{\frac{5}{4} m^{2} A_{1}^{(1)}+\frac{5}{3} M m A_{3}^{(1)}+\left(M^{2}+\frac{1}{2} m^{2}\right) A_{5}^{(1)}\right\} \\
\mathcal{E}^{2} A_{7}^{(2)}=\frac{7}{4}\left\{\frac{5}{36} m^{2} A_{3}^{(1)}+\frac{13}{25} M m A_{5}^{(1)}\right\}, B_{7}^{(1)}=\frac{49}{2}\left\{\frac{1}{12} m^{2} A_{3}^{(1)}+\frac{1}{5} M m A_{5}^{(1)}\right\} \\
\varepsilon^{2} A_{9}^{(2)}=\frac{99}{400} m^{2} A_{5}^{(1)}, B_{9}^{(2)}=\frac{81}{40} m^{2} A_{5}^{(1)}
\end{gathered}
$$

Thus, the cumulative solution is

$$
\rho^{(2)}(\sigma, \tau)=-\sum_{n=0}^{4} \frac{1}{8(2 n+1)^{3}}\left\{\begin{array}{l}
\left(2(2 n+1) A_{2 n+1}^{(2)}+\left[2(2 n+1)^{2} \sigma^{2}-1\right] B_{2 n+1}^{(2)}\right) \cos (2 n+1)(\sigma-\tau)+ \\
+\left(2(2 n+1) B_{2 n+1}^{(2)}-4(2 n+1)^{2} A_{2 n+1}^{(2)}\right) \sigma \sin (2 n+1)(\sigma-\tau)
\end{array}\right\}
$$

where the cumulative solution $\rho^{(2)}(\sigma, \tau)$ contains a quadratic term of $\sigma$. Of course, the solutions referring to the situations such as $l>1$ and $m_{1}>2$ as well as taking the sine term in equation (11) as the zeroth order solution etc. can be calculated similarly except that the amount of mathematical calculation will be too much.

Substituting equations (11), (14) and (17) into (7), we can get the perturbation-cumulative solutions up to an accuracy of the second order approximation, where the terms $\cos (2 n+1)(\tau-\sigma)$ and $\sin (2 n+1)(\tau-\sigma)$ are included. Obviously, when $n=0$, we can get the first subharmonic waves.

\section{Discussion}

In solving the problem of the elliptical drum, Mathieu [9] obtained a differential equation to describe the vibration of a membrane, of which the solutions so-called the Mathieu's functions were given by the singular perturbation method, where the terms of period-doubling vibrations and the harmonic vibrations of them were included. In this paper, however, we obtained the equation (4), which describes the propagation of the waves in the flowing media. In order to solve the equation, the cumulative solutions in the perturbation approximation were obtained, where the first subharmonics and the harmonic waves of them were included.

In order to observe the first subharmonic wave, the experiments $[6,7]$ were carried out in a tank, into which a sound wave at frequency $1.948 \mathrm{MHz}$ was transmitted. Only a sound wave with the same frequency was received when the water in the tank was static (cf. figure 1). On the other hand, if the medium was disturbed by a stick or a stirrer [6,7], an appreciable first subharmonic $(0.974 \mathrm{MHz})$ was observed (figure 2).

From the equations (11), (14) and (17), the first 
subharmonic wave and its odd order harmonics i.e. the period-doubling bifurcation solutions are obtained. As was mentioned in the beginning of this paper, the authors of Ref. [1] as well as Ref. [3] demonstrated that as the control parameter (s) increase, the system can route to chaos through period-doubling bifurcations. Furthermore, an experimental investigation in acoustic area was carried out by the authors of Ref. [2], where the acoustic cavitation noise in the water was measured and "a subharmonic route to chaos including period-doubling bifurcations up to $f / 8$ has been observed in experiments on acoustical turbulence." However, it is worth noting that in this experiment only one parameter (acoustic pressure or acoustic Mach number $m$ ) can be controlled. However, in this paper there are two parameters $M$ (flow Mach number) and $m$ (acoustic Mach number), which can control the route of the system to chaos. Thus, it is reasonable to expect that the results obtained by this paper would help people to insight the mechanism of the turbulence formation.

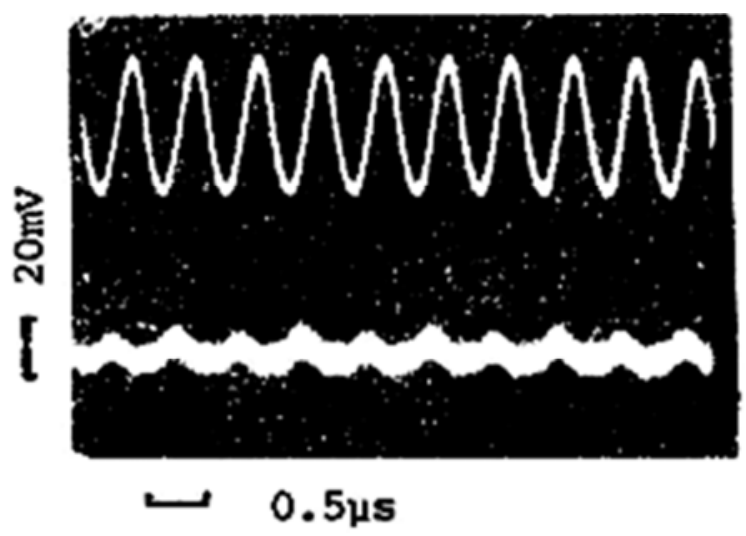

Fundamental frequency

Figure 1. The signals in static medium.

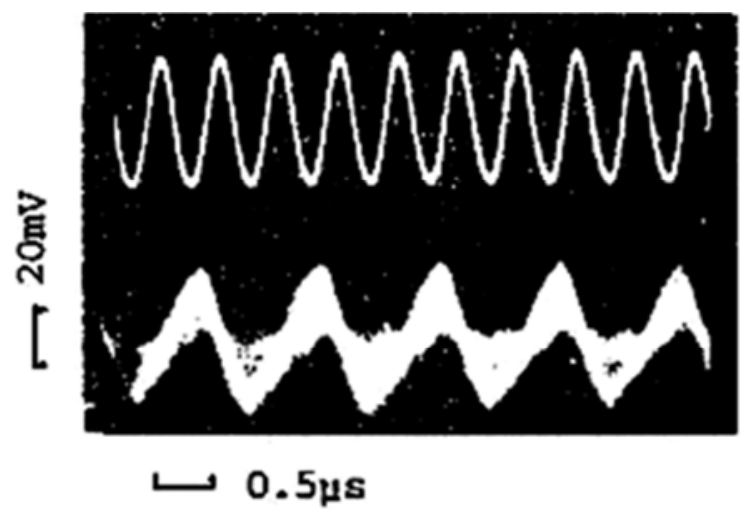

First subharmonic waves

Figure 2. The signals in disturbed medium.

\section{Conclusions}

Based on the Lighthill's equation, a new equation to describe the wave propagation in the disturbed medium was deduced and the so-called perturbation-cumulative approximation was suggested to solve this equation, from which the period-doubling bifurcation solutions (including the first subharmonic as observed in Refs. [6, 7]) were obtained. The results would help people to gain insights to the mechanism of the turbulence formation.

\section{Acknowledgements}

This research was supported by the National Natural Science Foundation of China (Grant No. 11274337).

\section{Appendix}

\section{Cumulative solutions}

From (12) and (15) the equations interested in us are as following

$$
\frac{\partial^{2} \rho(\tau, \sigma)}{\partial \tau^{2}}-\frac{\partial^{2} \rho(\tau, \sigma)}{\partial \sigma^{2}}=\left\{\begin{array}{l}
-A_{n}^{(1)} \cos n(\tau-\sigma) \\
-B_{n}^{(1)} \sin n(\tau-\sigma)
\end{array}=\left\{\begin{array}{l}
-\frac{1}{2} A_{n}^{(1)} e^{-i n(\tau-\sigma)}+c . c . \\
\frac{1}{2 i} B_{n}^{(1)} e^{-i n(\tau-\sigma)}+c . c .
\end{array}\right\}\right.
$$

If the solutions are periodic in time domain, that is

$$
\rho(\tau, \sigma)=\rho(\sigma) e^{-i n(\tau-\sigma)}
$$

then one has

$$
\frac{d^{2} \rho(\sigma)}{\partial \sigma^{2}}+n^{2} \rho(\sigma)=\left\{\begin{array}{l}
\frac{1}{2} A_{n}^{(1)} e^{i n \sigma}+c . c . \\
-\frac{1}{2 i} B_{n}^{(1)} e^{i n \sigma}+c . c .
\end{array}\right.
$$

Thus, the equation to be solved is

$$
\left[\frac{d^{2}}{d \sigma^{2}}+n^{2}\right] \rho(\sigma)=f(\sigma) e^{i n \sigma}
$$

where $A$ is a constant. Obviously, this equation has cumulative solutions, which can be solved by Lagrange parameter variation method [10]. Let

$$
\rho(\sigma)=D_{1}(\sigma) e^{i n \sigma}+D_{2}(\sigma) e^{-i n \sigma}
$$

Substituting (19) into (18) and assuming

$$
\frac{d D_{1}(\sigma)}{d \sigma} e^{i n \sigma}+\frac{d D_{2}(\sigma)}{d \sigma} e^{-i n \sigma}=0
$$

yield

$$
\frac{d D_{1}(\sigma)}{d \sigma} e^{i n \sigma}-\frac{d D_{2}(\sigma)}{d \sigma} e^{-i n \sigma}=\frac{1}{i n} f(\sigma) e^{i n \sigma}
$$

From (20) and (21)

$$
D_{1}(\sigma)=\frac{1}{2 \text { in }} \int f(\sigma) d \sigma
$$

and 


$$
D_{2}(\sigma)=-\frac{1}{2 i n} \int f(\sigma) e^{2 i n \sigma} d \sigma
$$

Thus, the cumulative solution can be denoted by

$$
\rho(\sigma)=e^{i n \sigma} \frac{1}{2 i n} \int f(\sigma) d \sigma-e^{-i n \sigma} \frac{1}{2 i n} \int f(\sigma) e^{2 i n \sigma} d \sigma
$$

\section{References}

[1] M. J. Feigenbaum, "Quantitative universality for a class of nonlinear transformations", J. Stat. Phys. 19, 25-52 (1978).

[2] W. Lauterborn and E. Cramer, "Subharmonic route to chaos observed in acoustics", Phys, Rev. Lett. 47, 1445-1448 (1981).

[3] Song-Yoon Kim and Bambi Hu, "Bifurcations and transitions to chaos in an inverted Pendulum", Phys. Rev. E. 58, 3028 (1998).

[4] T. B. Benjamin, F. Ursell, "The stability of the plane free surface of a liquid in vertical periodic motion", Proc. Roy. Soc. A 225, 505-516 (1954).

[5] Ruby Lawrence, "Applications of the Mathieu equation", Am. J. Phys. 64, 39-44 (1996).

[6] D. Shao, Z. W. Qian, "First subharmonic sound in disturbed water", Chinese Physical Letters 4, 133-135 (1987).

[7] Z. W. Qian and D. Shao, "Some interesting phenomena of first subharmonic of sound in water". In: Proc. IUPAP, IUTAM Symposium on Nonlinear Acoustics. V. K. Kidrinskii, editor. Vol. 2. Novosibirsh, Academy of Sciences USSR (1987), P. 245-248.

[8] M J. Lighthill. "On sound generated aerodynamically", Proc Roy Soc (London) A 211, 564-587 (1952).

[9] N W. McLachlan, Theory and application of Mathieu functions. (Dover, New York, Publications, 1964), pp, 1-401.

[10] Zu-Wen Qian, "Cumulative solutions of nonlinear longitudinal vibration in isotropic solid Bars", Chin. Phys. B, 23, 064301 (2014). 